\title{
Developmental biology in bioartificial tissue design: manufacturing and regulatory considerations
}

\author{
Petros Lenas*,1 \\ ${ }^{1}$ College of Science, Harbin Institute of Technology Shenzhen, HIT Campus, Shenzhen University Town, Xili, Shenzhen 518055, P. \\ R. China \\ * Author for correspondence: lenaspetros@yahoo.com
}

\begin{abstract}
“The structural robustness and high semi-autonomy in the progression of development makes developmental modules the ideal targets of tissue engineering"
\end{abstract}

First draft submitted: 29 August 2017; Accepted for publication: 2 November 2017; Published online: 26 January 2018 Keywords: bioartificial tissue $\bullet$ developmental biology $\bullet$ manufacturing $\bullet$ quality by design $\bullet$ regulation $\bullet$ tissue
engineering

\section{The properties of bioartificial tissues that can assure clinical efficacy remain undetermined}

Tissue engineering emerged as a distinct research field three decades ago with the goal to solve the problem of organ shortage for transplantations with biological tissue substitutes composed of cell-biomaterial (scaffolds) assemblies, bioartificial tissues. Although the clinical applications of bioartificial tissues with simple structure - such as: bladder, skin, cartilage, trachea and blood vessels - have been placed on track, the clinical translation in the case of tissues with complex structure and function - such as: liver, heart and kidney - proved to be a challenging problem in which no substantial progress has been yet achieved despite the intense research efforts and the sophisticated systems for the 3D cell culture developed in the field (e.g., micro-patterning, bioprinting, etc.). It has been said metaphorically that "we are able to fabricate constructs that 'look like tissue, smell like tissue and taste like tissue' but not some that also function equally like one", to denote that the properties that tissue engineering methods implement into bioartificial tissues are not these that are required for their functionality and consequently their clinical efficacy is not the expected one [1]. The determination of the properties of native in vivo tissues that bioartificial ones should have to be clinically efficacious, though was set as a priority from the beginning of the research activities in the field, as it was stated in the first issue of the journal of Tissue Engineering in 1995 [2], has not progressed, and tissue engineers are wondering "how good is good enough" (Butler D in [3]). It is only recently that the issue of clinical efficacy started to be addressed methodologically with the transition of tissue engineering approaches toward the exploitation of the mechanisms of in vivo tissue development which led to the rationalization of bioartificial tissue design but also of manufacturing and regulatory procedures based on concepts of developmental biology that offered new bioartificial tissue evaluation indices.

\section{Developmental biology inspired implants install in situ natural developmental processes}

In order to overcome difficulties in clinical translation, the tissue engineering research community proposed, in a workshop sponsored by the NIH of USA with the purpose to re-think research directions in the field, to rely on the mechanisms of the in vivo tissue development in order to make use of the inherent, programmed by evolution, capacity of cells to be self-organized to authentic tissue structures which could enhance the likelihood of clinical success [3]. Observations along a century of experimentation in embryology have concluded that some empirical concepts are of general applicability in the development of organisms and they can be used in the design of bioartificial tissues in a new methodology named 'developmental engineering' [4]. The most important empirical concept which argues in favor of the feasibility of such a developmentally relevant design, either in vitro for the fabrication of authentic tissue structures or in vivo for the induction of the in vivo-like developmental processes 
by implants, is the 'robustness' of development which confers stability to the process of tissue formation against environmental disturbances, reported for first time almost a century ago [5].

The robustness of development is related with the empirical concept of 'path-dependence' according to which each developmental stage sets the optimal conditions for the next one to take place, assuring therefore that development will progress on the natural developmental pathway. For example, during endochondral ossification (indirect bone formation through a cartilage template that takes place in the development of long bones of the body), optimal conditions for bone formation are established by the developing cartilage itself that provides osteoinductive and angiogenic signals, acting therefore as a self-generated optimal, intelligent scaffold that can orchestrate the processes involved in bone development [4]. The path dependence excludes the possibility of direct fabrication of final tissue forms in processes of one stage of cell seeding in scaffolds which was until recently the common practice in tissue engineering, and which, tissue engineers have characterized as 'perhaps naive' (Van Mow C in [3]) compared to the complexity of in vivo tissue development. As a tissue passes through several successive developmental stages, a gradual re-organization of the signaling pathways of its cells, with the modification of gene expression, takes place which continuously updates the cell properties. This re-organization allows the cells to have at each stage the properties needed to interact, move and spatially self-organize to the appropriate intermediate tissue structures that when are gradually reformed (with the parallel updating the signaling pathways and cells properties) they can be finally transformed to mature tissue structures. For some intermediate tissue forms appearing during tissue development, called 'developmental modules', the previous stages have equipped them with the operation of signaling pathways that can take over the further development without the need for additional external to developing tissue signals. Developmental modules, such as limb buds, tooth germs, mesenchymal cell condensations and the growth plate cartilage template in endochondral ossification, constitute integral units exhibiting internal coherence among their cells and a high degree of independence from other co-developing tissues so that they are able continue their development even ectopically [6].

The structural robustness and high semi-autonomy in the progression of development makes developmental modules the ideal targets of tissue engineering instead of any other type of 3D distribution of cells in scaffolds which, as irrelevant to a developing tissue form, lacks these properties and it has therefore a structure that is unstable against environmental disturbances and unable to be re-organized by itself (without external instructions) to the final authentic tissue structure [4]. The recent use of developmental modules as bioartificial tissue implants led to considerable progress in the methodological evaluation and prediction of clinical efficacy based on information from developmental biology. For example, developmentally inspired constructs of hypertrophic chondrocytes (the terminal stage of differentiation in the chondrogenic cell lineage) in scaffolds, when implanted ectopically, operated as the in vivo cartilage templates of indirect bone formation triggering autonomously (in the absence of any instructive external signals) the whole phenomenon of endochondral ossification and leading to the formation of functional bone tissue [7]. Developmental modularity became therefore the first similarity index between bioartificial and real tissues related with clinical efficacy and its adoption by the tissue engineering has been characterized as an important milestone in the field because it renders the bioartificial tissue design rational [8].

\section{Developmental biology rationalizes manufacturing \& regulatory procedures}

Properties of products that contain cells and biomaterials, either externally added or produced by the cells, are susceptible to changes in manufacturing process, as are also the biologics (e.g., recombinant proteins). Such products cannot be easily and fully characterized in the laboratory as the conventional drugs made by chemical synthesis. This led to the adoption by regulatory authorities of the policy 'the product is the process' [9]. From the other side however, it can be also said that 'the process is the product' [10]. Indeed, a bioartificial tissue that is not related to a developing tissue form can have arbitrary properties concerning the cell composition, spatial arrangement or differentiation state, as for instance a bioartificial bone implant for long bone defects that is made by the direct osteogenic differentiation of stem cells on scaffolds. Since no such tissue form exists in the in vivo development of long bones, which takes place with the indirect bone formation through a cartilage template made by chondrogenic (instead the osteogenic) differentiation of stem cells, this product cannot be uniquely defined and therefore it can neither determine a unique in vitro process because of the lack of the in vivo counterparts as reference product and process. For example, there could not be any information from developmental biology concerning the optimal stage of the osteogenic differentiation of stem cells that has to be achieved in vitro before the implantation of such a bioartificial construct with osteoblasts on a scaffold to treat long bone defects, and therefore an in vitro process that can provide authentic bone tissue structures that could assure the clinical efficacy cannot be determined. 
Consequently, in lack of a well-defined and scientifically grounded on developmental biology (which provides the only examples of tissue formation) process, a bioartificial tissue product not related to any developing tissue form cannot be re-designed to achieve closer structural similarity with the real tissue that could lead to better clinical performance. In a nondevelopmental process, no scientific predictions can be made on how the process parameters influence the product properties that are related with the clinical efficacy, in other words, the process lacks controllability and it cannot be therefore modified rationally. If, for instance, the implantation of a scaffold with osteoblasts for long bone defects does not lead to satisfactory bone formation, it cannot be concluded that the osteoblasts need to be in a more or less advanced differentiation stage because no such process exists in vivo for comparison. The product re-design was a critical problem in tissue engineering companies and it had not escaped the attention of business world which referred to this very early. Business analysts had mentioned two decades ago that re-engineering a candidate product to improve its properties was a very difficult problem that resulted to a high risk of failure in clinical product development in tissue engineering companies (Arrow AK in [11]), a prophetic comment since 4 years later two pioneering companies of the field, Organogenesis and Advanced Tissue Sciences, declared bankruptcy [12].

The establishment of properties of developmental modules into bioartificial tissues improved radically this problematic situation. For instance, instead of attempting to fabricate bioartificial bone in a nondevelopmental process, tissue engineers have started using the methodology of developmental engineering which suggests the fabrication of cartilage templates with the chondrogenic differentiation of stem cells on scaffolds, which, as implants, can install in situ the natural process of endochondral bone formation [7]. The major advantage of this, inspired from developmental biology, approach is that it permits the evaluation of the implant by comparison with the real in vivo cartilage template of endochondral ossification. In this case, well-informed from developmental biology, rational modifications in the design of the implant (e.g., cell composition, differentiation state and pattern of spatial arrangement) can be made to bring it closer to the in vivo cartilage template with the appropriate modifications of the in vitro process which is uniquely defined to fabricate a specific product with well-defined properties, i.e., the developmental module of the cartilage template as this is described in numerous studies of developmental biology. For instance, Scotti et al. have optimized the cartilage templates to induce in situ, after ectopic implantation, the endochondral bone formation by differentiating in vitro human mesenchymal stem cells on scaffolds to chondrocytes up to the late stage of hypertrophy [7]. According to the authors, implants with chondrocytes in earlier differentiation stages had not optimal performance in inducing bone formation in accordance with information of developmental biology about the in vivo endochondral ossification.

It comes out therefore that processes for the fabrication of bioartificial developmental modules, instead of any developmentally irrelevant bioartificial tissue form, and their corresponding products (developmental modules), can be rigorously characterized and rationally re-designed to optimize clinical efficacy in accordance to information from developmental biology. Such a methodological, based on developmental biology, way of process and product design complies with the regulatory concept of 'Quality by Design' that the US FDA and the EMA in collaboration with FDA, have adopted for pharmaceutical manufacturing [13,14]. According to this concept, developed by the engineer and management consultant Juran in 1986 [15], "quality cannot be tested into products, i.e., quality should be built in by design" [16]. In the case of arbitrary bioartificial tissue forms that are not related with the in vivo tissue development, the quality of the product, for example, clinical efficacy and safety, cannot not be built in by design because it is unknown which properties they should have in order to induce after implantation the natural processes of tissue formation instead of any other undesirable abnormal one. Contrary to the 'Quality by Design' concept, the clinical efficacy in the case of such bioartificial tissues is tested after implantation with great difficulty to re-design their properties with trial-and-error approaches to achieve better performance. As Niklason L mentioned, in lack of a rational methodology, tissue engineering had adopted the 'tissue try this' approach, (a phrase coined by Shannon Dahl) [3]. Similarly, Ingber DE noted, that the research practice in tissue engineering was diminished to "put together your best ideas and materials, throw them in an animal, and pray for the best" which offered "ittle understanding of why something did or did not work" [3]. From the other side however, in the new methodology of tissue engineering which is based on developmental biology, developmental modularity, as a property of bioartificial tissues, allows the prediction of clinical efficacy by reference to the in vivo developmental mechanisms executed by developmental modules as these mechanisms are described in developmental biology. Since modularity allows the progression on development with high semi-autonomy, it can also take place in vitro, which permits an in vitro experimentation to determine optimal conditions and timing for implantation as the necessary conditions for the induction of the desired natural developmental processes and therefore it increases the chances to achieve the best results in vivo after 
implantation accelerating in this way the clinical translation but also contributing to the assurance of the safety of implants [17].

Further facilitation of the clinical translation can be achieved, thanks to the robustness of development. An in vitro process for the formation of bioartificial developmental modules (to be used as implants to install in situ natural developmental processes), which is similar to the corresponding in vivo process, exhibits the same robustness to environmental disturbances, that are abundant in vitro, and therefore it can be scaled-up for largescale production for clinical trials and subsequent manufacture of the clinical product. The robustness of such an in vivo-like developmental process offers high reproducibility in the process and consequently in the end product (bioartificial tissue) properties. Contrary to such in vivo-like processes that are self-controlled, nondevelopmental processes do not have inherent robustness and require sophisticated process control and automation. The lack of process robustness for large-scale production can result to the failure of a product to enter the clinics despite the research and development efforts. As Naughton G, President of Advanced Tissue Sciences Inc., commended, after the bankruptcy of the company at the turbulent times for tissue engineering at the beginning of 2000 [12], the margins were poor because the manufacturing process for bioartificial skin was not reproducible [18]. According to Naughton, the lack of reproducibility from batch to batch increased the cost of the production. The process had not any similarity with the in vivo skin development, consisting of neonatal fibroblasts cultured on a bioabsorbable mesh, and there was therefore no reason to be inherently robust.

The above considerations related with the manufacturing and regulatory importance of developmentally relevant bioartificial tissue forms are especially critical at this time because a growing number of publicly funded centers have been created worldwide with the purpose of supporting the transition from early research to late clinical trials and commercial supply providing guidance to industry for manufacturing according to regulations [19].

\section{Future perspective}

The proposition of the use of developmental mechanisms in tissue engineering [3] and the subsequent analysis of the concepts of developmental biology, such as robustness, modularity, path-dependence, that should be employed for the practical implementation of this approach [4], have motivated tissue engineers to attempt to use these concepts in the experimental design for various bioartificial tissues. However, most of the efforts until now have focused on bioartificial bone because of sufficient information provided by developmental biology about the in vivo process of endochondral ossification and the properties of developmental modules appearing in this (cartilage template with hypertrophic chondrocytes, mesenchymal stem cell condensations). The major obstacle therefore for a wide use of this approach in several tissues is the lack of information in developmental biology concerning developmental modules. Although developmental modularity was the dominant paradigm in embryology, it was later replaced by a genetic approach dealing with cell differentiation that could be easily examined with the existing biochemical techniques at that time, to return recently as a favorite subject of studies in systems biology that has the appropriate concepts and methods to deal with properties established in biological systems by their component interactions as such modularity is [6]. According to studies of systems biology, the autonomy of developmental modules is due to the autonomous dynamics of gene-protein interaction networks established throughout their cells from cell-to-cell interactions that, without external factors, activate the necessary signaling pathways for the continuation of development [20]. Computational models of the gene-protein interaction networks that confer autonomy on macroscopic multicellular developmental modules are robust and converge, for a large variety of initial conditions, such as initial gene expression, and parameters, such as kinetic constants, to the same solution that corresponds to the spatially differential gene expression observed in the cellular patterns of developmental modules [20]. A mathematical and computational analysis, therefore that connects the gene-protein interaction network activated inside an in vitro developing tissue form with the process parameters (e.g., concentration and diffusion of signaling molecules inside a scaffold) will allow the computational design of bioartificial tissues with the selection of in vitro conditions that restore the cell-to-cell communication that establishes such networks advancing the bioartificial tissue evaluation indices from the cell level (e.g., cell viability and growth) to a multicellular tissue level (new global properties arising from what all the cells do together). As tissue engineers have commented for the advantages of developmental engineering, modelling approaches directed to global properties indicative of capacity to progress in developmental processes, will permit the evaluation of explicit hypotheses that would inform process design advancing tissue engineering from a trial-and-error empirical endeavor to a rigorous technology with modifiable design parameters as the other branches of engineering [21]. 


\section{Financial \& competing interests disclosure}

The author has no relevant affiliations or financial involvement with any organization or entity with a financial interest in or financial conflict with the subject matter or materials discussed in the manuscript. This includes employment, consultancies, honoraria, stock ownership or options, expert testimony, grants or patents received or pending, or royalties.

No writing assistance was utilized in the production of this manuscript.

\section{References}

1 Polykandriotis E, Popescu LM, Horch RE. Regenerative medicine: then and now - an update of recent history into future possibilities. J. Cell. Mol. Med. 14(10), 2350-2358 (2010).

2 Nerem RM, Sambanis A. Tissue engineering: from biology to biological substitutes. Tissue Eng. 1(1), 3-13 (1995).

3 Ingber DE, Mow VC, Butler D et al. Tissue engineering and developmental biology: going biomimetic. Tissue Eng. 12(12), 3265-3283 (2006).

4 Lenas P, Moos M, Luyten FP. Developmental engineering: a new paradigm for the design and manufacturing of cell-based products. Part I: from three-dimensional cell growth to biomimetics of in vivo development. Tissue Eng. Part B Rev. 15(4), 381-394 (2009).

5 Waddington CH. Canalization of development and the inheritance of acquired characters. Nature 150(3811), 563-565 (1942).

6 Gilbert SF, Opitz JM, Raff RA. Resynthesizing evolutionary and developmental biology. Dev. Biol. 173(2), 357-372, (1996).

7 Scotti C, Tonnarelli B, Papadimitropoulos A et al. Recapitulation of endochondral bone formation using human adult mesenchymal stem cells as a paradigm for developmental engineering. Proc. Natl Acad. Sci. USA 107(16), 7251-7256 (2010).

8 Martin I. Engineered tissues as customized organ germs. Tissue Eng. Part A 20(7-8), 1132-1133 (2014).

9 EudraLex. The rules governing medicinal products in the European Union, volume 4, EU guidelines for good manufacturing practice for medicinal products for human and veterinary use. Annex 2: Manufacture of biological active substances and medicinal products for human use. European Commission (2012). http://ec.europa.eu/health/files/eudralex

10 Thompson K.Commentary: The final piece of the jigsaw. Why the UK is building cell therapy capacity. MedNous (2014). www.mednous.com/sites/default/files/CommentaryKeithThompsonApr14.pdf

11 Stone A. Investing in tissue engineering. Business week, July 27, 1998. www.bloomberg.com/news/articles/1998-07-27/investing-in-tissue-engineering

12 Bouchie A. Tissue engineering firms go under. Nat. Biotechnol. 20(12), 1178-1179 (2002).

13 FDA. Final Report. Pharmaceutical CGMPs for the 21st century: a risk-based approach (2004). www.fda.gov/downloads/drugs/ developmentapprovalprocess/manufacturing/questionsandanswersoncurrentgoodmanufacturingpracticescgmpfordrugs/ucm 176374.pdf

14 EMA and FDA extend pilot program for parallel assessment of quality-by-design applications (2014). www.ema.europa.eu/ema/index.jsp?curl=pages/news_and_events/news/2014/03/news_detail_002035.jsp\&mid=WC0b01ac058004d5c1

15 Juran JM. The quality trilogy: a universal approach to managing for quality. Quality Progress 19(8), 19-24 (1986).

16 FDA Guidance for Industry: Q8(R2) Pharmaceutical development. Revision 2 (2009). www.fda.gov/downloads/drugs/guidances/ucm073507.pdf

17 Fisher JN, Peretti GM, Scotti C. Stem cells for bone regeneration: from cell-based therapies to decellularised engineered extracellular matrices. Stem Cells Int. 2016, 9352598 (2016).

18 Leventon W. Synthetic skin. IEEE Spectrum 39(12), 28-33 (2002).

19 Brindley DA, Reeve BC, Sahlman WA et al. The impact of market volatility on the cell therapy industry. Cell Stem Cell 9(5), 397-401 (2011).

20 von Dassow G, Meir E, Munro EM, Odell GM. The segment polarity network is a robust developmental module. Nature 406(6792), 188-192 (2000).

21 Yang G, Rothrauff BB, Tuan RS. Tendon and ligament regeneration and repair: clinical relevance and developmental paradigm. Birth Defects Res. C Embryo Today 99(3), 203-222 (2013). 